\title{
Kommentar
}

Schweiz. Zschr. GanzheitsMedizin 17, 141-144 (2005). @ Verlag für GanzheitsMedizin, Basel. www.ganzheitsmedizin.ch

\section{Margarethe Combé}

\section{Arteriosklerose - ein komplexes Geschehen}

\author{
In-vivo-Studie zur ganzheitlichen Wirkung von Padma 28
}

$D_{k}^{\text {ie }}$ ie häufigste entzündliche Erkrankung in der westlichen Welt ist die Arteriosklerose, eine chronische Krankheit, die bereits im Fetalleben beginnt; während Kindheit und Adoleszenz schreitet der Prozess langsam voran, beschleunigt sich dann aber beim Erwachsenen, was zu fatalen Folgen wie Plaqueruptur und Infarkt führen kann. Autopsiebefunde junger und klinisch unauffälliger Menschen bestätigten bereits fortgeschrittene und obstruktive Gefässläsionen. Bei «gesunden» Herzspendern zwischen 20-29 Jahren wurde in bis zu $37 \%$ der Fälle eine beachtliche Ausdehnung einer Koronarsklerose gefunden; zwischen $30-39$-Jährigen waren es $60 \%$, bei über 50-Jährigen sogar 85\% [2]. So verwundert es eigentlich nicht, dass die initiale Manifestation der kardiovaskulären Erkrankung bei der Hälfte der Patienten Sekundentod oder Myokardinfarkt sind. Diese Fakten verdeutlichen die Notwendigkeit, das Geschehen besser zu verstehen, um frühzeitig in die Entwicklung der Arteriosklerose eingreifen zu können.

Die Hypercholesterinämie scheint Hauptakteur bei der Entstehung arteriosklerotischer Schäden und der Progression des Leidens zu sein; unter diesen pathophysiologischen Aspekten sind die Low-densitiy-Lipoproteine (LDL) die bedeutendste Fraktion im Blut.

\section{LDL Cholesterin - eine lebensnotwendige Substanz}

Die LDL-Struktur ist in den letzten 20 Jahren weitgehend aufgeklärt worden [4]. LDL sind kugelförmige Gebilde, die unterschiedliche Lipide an der Ober- fläche und im Kernbereich enthalten. An der Oberfläche befinden sich Phospholipide und freies Cholesterin sowie ein Teil des Apolipoprotein-B-Moleküls, das für die Interaktion mit dem Rezeptor benötigt wird. Der grösste Teil des Apolipoprotein-B-Moleküls eines der grössten bekannten Proteine - taucht in den Kern des Partikels ein, wo es sich mit den dort vorhandenen Cholesterinestem und Triglyceriden verbindet. Jedes LDL-Partikel trägt nur ein Apolipoprotein-B-Molekül, das unter genetischen Gesichtspunkten von Vater und Mutter her stammen kann. Somit gibt es prinzipiell eine gemischte LDL-Population im Kreislauf. Die Funktion der LDL besteht zum einen in der Versorgung peripherer Gewebe mit Cholesterin. Zum anderen spielen LDL auch eine bedeutende Rolle in der Versorgung der Körperzellen mit essentiellen Fettsäuren (Linolsäure), da Cholesterinester einen hohen Anteil an diesen Fettsäuren aufweisen. Weiterhin transportieren die LDL verschiedene lipophile Substanzen, unter anderem Carotinoide und Vitamin E [4].

\section{LDL Subpopulation}

Im Elektronenmikroskop haben LDL einen Durchmesser von 21 und $24 \mathrm{~nm}$. Durch Ultrazentrifugieren können LDL in zwei grössere Subpopulationen unterteilt werden, die als LDL 1 und LDL 2 bezeichnet wurden. LDL 1, auch IDL (intermediate density lipoprotein) genannt, werden bei einer Dichte zwischen 1,006 und 1,019g/ml isoliert, die quantitativ bedeutenderen LDL 2 zwischen 1,0919 und 1,063g/ml [4]. Mit speziellen Subfraktionierungsverfah- ren kann man bis zu 6 einzelne Unterg ruppen der LDL isolieren. Dem Verhalten in der Elektrophorese entsprechend wurden die LDL auch als BetaLipoproteine bezeichnet. In der Lipoprotein-Elektrophorese sind IDL und LDL nahezu untrennbar, ausserdem ist eine relativ dichte Fraktion der Verylow-density-Lipoproteine (VLDL) ebenfalls mit Beta-Mobilität ausgestattet. Unter pathophysiologischen Gesichtspunkten hat sich noch eine weitere Unterteilung der LDL durchgesetzt, nämlich in «big-light» und «small-dense»LDL. In Bezug auf die Atherogenese kommt auf Grund mehrerer Untersuchungen der «small dense»-LDL-Fraktion eine wesentliche Bedeutung $\mathrm{zu}$ [4].

Die durchschnittliche LDL Cholesterinkonzentration steigt mit zunehmendem Lebensalter stetig:

- 20-jährige Männer haben Durchschnittswerte von $110 \mathrm{mg} / \mathrm{dl}$

- 50-jährige Männer haben Durchschnittswerte von $160 \mathrm{mg} / \mathrm{dl}$ [4].

Bei Frauen findet sich zwischen dem 20. und 40. LJ nur ein geringer Anstieg von 110 auf $130 \mathrm{mg} / \mathrm{dl}$; mit Beginn der Menopause steigen die Werte dann deutlich an. Offensichtlich spielt die Hormonsituation eine bedeutende Rolle! Geht ein Gefässrisiko auch im fortgeschrittenen Alter der Frau von erhöhten Cholesterinwerten aus? Diese Frage konnte bisher nicht eindeutig b e a n twortet werden. Sind es überhaupt die absoluten Cholesterinwerte, von denen ein solches Gefahrenpotential ausgeht? Zwei vergleichbare Fälle relativieren diese Hypothese: Ein 3-jähriger Knabe mit familiärer Hypercholesterinämie starb durch einen Myokardinfarkt, während eine 33-jährige Frau mit der gleichen Genmutation für den LDL-Rezep- 
tor und der gleichen Plasma-Cholesterolkonzentration $(800 \mathrm{mg} / \mathrm{dl})$ an e i n e r nichtkoronaren Ursache verstarb [2].

\section{LDL und Arteriosklerose- Risiko}

Der Prozess der Atherogenese wird durch die direkte Aufnahme und Ablagerung von LDL-Partikeln im subintimalen Raum der Arterie eingeleitet und unterhalten. Interaktionen mit der Gefässwandmatrix halten die Partikel dort fest und LDL-modifizierende Abbauprodukte verursachen eine deutliche lokale Entzündungsreaktion mit initialer Aktivierung von Makrophagen, T-Lymphozyten und glatten Muskelzellen. Dies führt zur Phagozytose von LDL-Partikeln, wobei die meisten Bestandteile des LDL wie das Apolipoprotein B, Phospholipide und Triglyceride mobilisiert und entfernt werden können. Das Cholesterin dagegen bleibt in der Gefässwand, teilweise als Cholesterinester und teilweise als freies Cholesterin, liegen. Letztendlich ist es also der Cholesterinanteil des LDL, welcher zur Plaquebildung führt. Die Aufsättigung der Endothelzellen mit Cholesterin und die nun einsetzenden Oxidationsprozesse führen zu einer nachlassenden Stickstoffmonoxyd( $\left.\mathrm{NO}^{-}\right)$Synthese, die sich auf die Gefässspannung negativ auswirkt und somit zu einer endothelialen Dysfunktion führt [4]. Denn $\mathrm{NO}^{-}$bewirkt nicht nur eine Vasodilatation, sondern auch einen wirksamen atherosklerotischen Schutz, der Thrombozytenaggregation und Proliferation glatter Muskelzellen verhindert und Lipidperoxidation und Expression von Adhäsionsmolekülen vermindert. In diesem Zusammenhang ist die nun einsetzende zunehmende Produktion von «reactive oxygen species» (ROS) in den Gefässen entscheidend. Denn Superoxid reagiert rasch mit $\mathrm{NO}^{-}$, was zur Bildung von Peroxidanion und weniger $\mathrm{NO}^{-}$-Bioverfügbarkeit führt. Neuere Untersuchungen zeigten, dass ROS, speziell Peroxinitrit, Tetrahydrbiopterin oxidieren kann, was ein kritischer Cofaktor für die Nitritoxidsynthase darstellt [6]. Vice versa kann die epitheliale Dysfunktion durch verschiedene nicht verwandte
Antioxidantien einschliesslich membranpermeabler Superoxid Dismutase, P robucol (Lipidsenker, Antioxidans), Vitamin C und Glutathion teilweise rückgängig gemacht werden [6].

ROS fördert ausserdem die Lipidperoxidation, stimuliert das Wachstum der glatten Muskelzellen und initiiert die Expression proinflammatorischer Gene [6]. Es aktiviert die MatrixMetallproteasen, die die Plaque instabil machen und zu Rupturen führen.

\section{Arteriosklerose, eine Autoimmunkrankheit ?}

All diese Untersuchungen beweisen, dass die Entstehung der Arteriosklerose ein zu komplexes Geschen ist, um es lediglich mit der Elimination eines «Zuviel» an LDL behandeln zu wollen. $\mathrm{Zu}$ viele Faktoren spielen eine Rolle und dominieren in verschiedenen Individuen ganz unterschiedlich. Traditionelle Risikofaktoren sind Hypercholesterinämie, Rauchen, männliches Geschlecht, Bluthochdruck, Diabetes mellitus und fortgeschrittenes Alter. Aber die «nicht traditionellen» Ursachen sind mindestens genau so entscheidend: Das Immunsystem, das die Arteriosklerose moduliert. Angeborene und erworbene Immunantworten reagieren auf Antigene in arteriosklerotischen Läsionen und beeinflussen die Entwicklung der Läsion. Viele bei der Entstehung der Arteriosklerose beteiligten Immunreaktionen sind auch Immunantworten auf andere endogene und exogene Pathogene [2]. Es ist bekannt, dass die Immunantwort im arteriosklerotischen Geschehen auch an ähnlichen Krankheitsprozessen mit Infektionen oder akuten und chronischen Krankheiten beteiligt ist. Die Entzündung ist grundsätzliche Voraussetzung für Beginn und Fortschreiten der Erkrankung; sie ist auch an Plaquerupturen und Thrombosen beteiligt. Die Reaktion des Immunsystems muss also im Kontext der Immunantwort auf die entzündlichen Komponenten der Arteriosklerose betrachtet werden.

Immunhistologische Untersuchungen arteriosklertischer Plaques haben gezeigt, dass sich vor allem T-Lymphozyten und Makrophagen in der Arte- rienwand anreichern als Antwort auf ein Pathogen. Bei der Arteriosklerose ist das Pathogen minimal oxidiertes LDL, in Spätformen oxidiertes LDL (oxLDL). OxLDLrepräs entierteines der bestcharakterisierten Autoantigene der Arteriosklerose; es exponiert an seiner Oberfläche Phospholipide, die in ihrer molekularen Struktur eng mit der Membranstruktur apoptotischer Zellen oder mit gewissen Mikroorganismen (z.B. streptococcus pneumoniae) verwandt sind. OxLDL dringt, begünstigt durch die endotheliale Dysfunktion, in die Gefässwand ein und wird von Makrophagen über ScavengerRezeptoren aufgenommen. Makrophagen können Antigene aus oxLDL an TLymphozyten präsentieren. Die T-Lymphozyten beginnen Zytokine zu produzieren, die die endotheliale Dysfunktion unterhalten. Zudem sezernieren sie mesenchymale Wachstumsfaktoren und Metallproteinasen, die die Proliferation und Migration von glatten $\mathrm{M}$ u skelzellen begünstigen. Autoantikörper gegen oxLDL werden bei Patienten mit Arteriosklerose nachgewiesen. Und TLymphozyten, die aus arteriosklerotischen Plaques kloniert wurden, proliferieren spezifisch gegen solche Makrophagen, die mit oxLDL beladen wurden [3]. Kürzlich konnte nachgewiesen werden, dass bei Patienten mit akutem Koronarsyndrom zirkulierende T-Lymphozyten vorhanden sind, die Gefässendothelzellen in vitro lysieren.

Somit sind die drei Kriterien erfüllt, die diese Entzündungsreaktion als Autoimmunkrankheit klassifizieren [3].

1. Antikörper oder Leukozyteninfiltrate müssen in pathologischen Läsionen vorhanden sein.

2. Autoantikörper oder autoreaktive Leukozyten müssen vorhanden sein.

3. Autoantikörper oder autoreaktive Leukozyten müssen nachweislich einen Gewebeschaden verursachen. Beim Menschen kann dieser Nachweis einer transplazentaren Übertragung, durch den adoptiven Transfer in ein Tiermodell oder durch Zell- oder Gewebeschädigung im Zelloder Organkulturmodell erfolgen [3]. Das Verständnis dieser pathophysiologischen Mechanismen gibt uns die Möglichkeit zu neuen therapeutischen Ansätzen. 

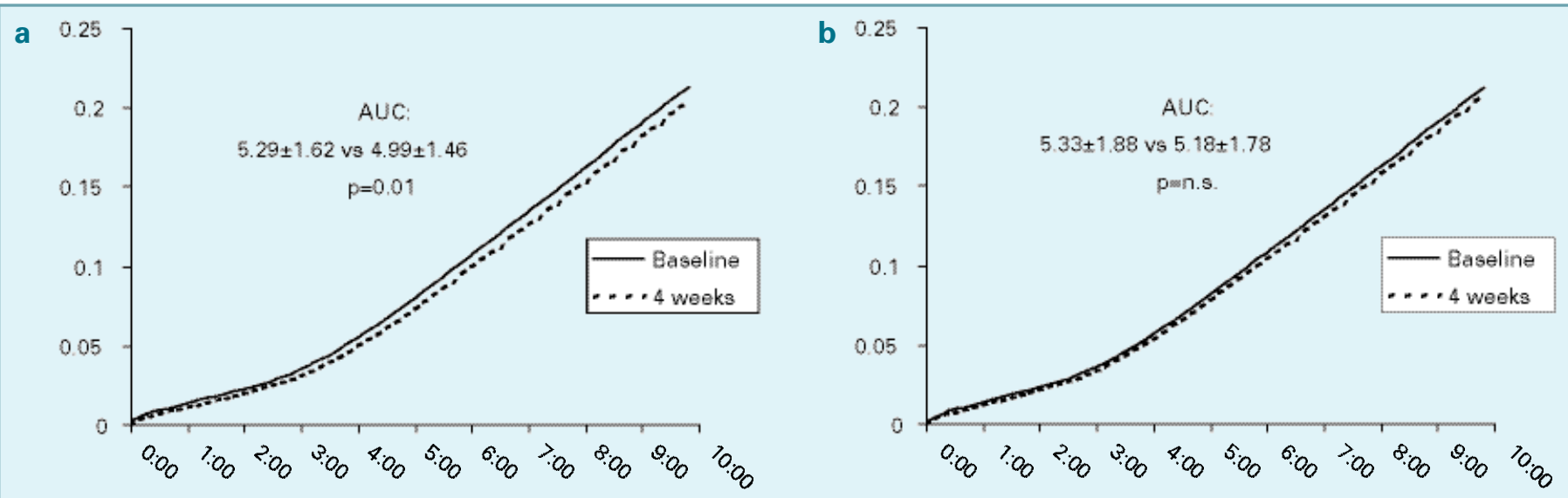

Abb. 1. Blutlipid-Oxidierbarkeit vor und nach 4 Wochen Einnahme von a) PADMA 28 bzw. b) Placebo. Die Verminderung der Blutlipid-Oxidierbarkeit ist nur bei der Verum-Gruppe statistisch signifikant $(p=0.01)$.

\section{Therapie}

LDL steht also unter den Faktoren, die zur Entwicklung der Arteriosklerose beitragen, an oberer Stelle. Zur Therapie wird traditionell die Senkung des LDL angestrebt.

\section{Diät}

Einen positiven Effekt haben Linolsäure und ölsäurereiche Fette und Öle. Die darin enthaltenen Omega-3-Fettsäuren hemmen die VLDL-Sekretion. Sie hemmen die Thromboxansynthese und stimulieren die Produktion von Prostazyklin PG13, wirken also antiaggressorisch und antiadhäsiv. Zusätzlich wird auch Leukotrien B4 vermindert.

Omega-3-Fettsäuren senken den Blutdruck und verbessern möglicherweise die Vollblutviskosität und die Deformierbarkeit der Erythrozyten. [4].

\section{Antioxidantien}

Natives LDL ist relativ harmlos. Erst durch die Oxidation wird es $\mathrm{zu}$ einem krankmachenden Faktor. Durch Interaktion von Endothelzellen, Makrophagen und glatten Muskelzellen wird es zum «minimal modified LDL» oxydiert. Dieses induziert die Bildung des Monozyten-chemotaktischen Proteins I und der Granulozyten- und Makrophagen-Koloniestimulierenden Faktoren. Dadurch werden Monozyten zu Makrophagen, durch deren Tätigkeit LDL weiterer Peroxidation unterliegt. Scavenger-Rezeptoren der Makrophagen erkennen oxLDL und phagozytieren es. LDL kann nicht in unkontrollierter
Menge aufgenommen werden; dagegen fehlt beim oxLDL der FeedbackMechanismus der LDL-Rezeptoren, so dass eine unkontrollierbare Phagozytose stattfindet. Es entstehen Schaumzellen [5]. OxLDL hemmt die günstige Wirkung des $\mathrm{NO}^{-}$; nach Gabe von Antioxidantien kann die hemmende Wirkung teilweise wieder aufgehoben werden. Vielleicht gibt es sogar eine eigenständige positive Wirkung von Antioxidantien auf die Gefässwand. [5] Endothelzellen, die mit Antioxidantien inkubiert worden waren, haben eine grössere Resistenz gegenüber den zytotoxischen Effekten des oxLDL bewiesen.

Seit Jahrzehnten wird in diesem Zusammenhang die Wirkung von Vitamin A, C, E und Beta Carotin diskutiert. Diese antioxidativ wirkenden Substanzen können wahrscheinlich die Umwandlung von LDL in oxLDL durch Reduktion der ROS beeinflussen. Eine antioxidative Therapie verbessert erfahrungsgemäss die Arteriosklerose beim Menschen [6]; idealerweise würde dadurch die Plaquebildung verlangsamt. In vitro lässt sich menschliches LDL oxidieren; nach Gabe von Antioxidantien ( $\alpha$-Tocopherol) kam es zu einer deutlichen Resistenz [5]. Vitamin E alleine zeigte allerdings in vivo kaum einen positiven Einfluss, da es in Lipidmembranen und Lipoprotein konzentriert ist und sich viele, gerade für die Arteriosklerose ausschlaggebende oxidative Prozesse im Zytoplasma und im Extrazellulärraum abspielen. Ausserdem wird Vitamin E nach Scavanging eines Radikals zum Tocopherolradikal, das unter Umständen die Lipidperoxidation steigert [6]. Das Topcopherolradikal kann durch andere Antioxidantien wie Vitamin C oder Coenzym Q 10 regeneriert werden. Es erscheint also sinnvoll, immer einen Cocktail von Antioxidantien zu geben. Es scheinen auch Einflüsse auf den Gefässtonus eine Rolle zu spielen. Antioxidantien verhindern thrombotische Prozesse am Gefässendothel, die letztlich auslösender Faktor für ein klinisches koronares Ereignis sind.

Flavonoide in Obst, Gemüse, Wein oder Tee scheinen einen positiven Effekt auf die KHK zu haben, was das «französische Paradoxon», also eine niedrige KHK-Inzidenz trotz fettre icher cholesterinreicher Ernährung bei reichlicher Zufuhr von im Rotwein enthaltenen Flavonoiden, erklären würde. Offenbar kann mit adäquaten Antioxidantien in adäquater Dosierung eine antiatherogene Wirkung erzielt werden. Insbesondere erzielte die Behandlung mit hochdosierten Antioxidantien in einer placebo-kontrollierten Doppelblindstudie an Patienten mit hohem oxidativem Stress sehr gute Resultate [12]. Der Zusammenhang zwischen der Oxidierbarkeit von LDL-Cholesterin und ischämischem Hirnschlag [13] bzw. Progress der Arteriosklerose [14] zeigt, dass die Oxidierbarkeit von LDL diagnostisch bedeutsam ist für den Verlauf der Erkrankung. So konnte VASSALLE et al. [15] eine Relation zwischen verminderter totaler antioxidativer Potenz (PAO) und Ausmass und Schweregrad koronarer Arteriener- 
krankungen (CAD) nachweisen [15].

\section{Immunmodulation}

Es scheint, dass die autoimmune Beschleunigung der Arteriosklerose durch verschiedene autoantigene Stimuli verursacht wird und das Ergebnis von multiplen humoralen und zellulären Entzündungsreaktionen ist. Bei vielen Patienten kann die Krankheit mit Antibiotika oder aber mit Statinen b e h a ndelt werden. Letztere vermindern MHC class II genauso wie die Expression von Adhäsionsmolekülen der Zellwand [7]. So überrascht es nicht, dass dieser Prozess sehr effektiv durch Immunmodulation beeinflusst werden kann [7].

\section{Padma 28}

Das pflanzliche Arzneimittel PADMA 28 wird seit vielen Jahren erfolgreich bei Arteriosklerose und arteriosklerotisch bedingten Symptomen wie z.B. Durchblutungsstörungen eingesetzt. Das in der Schweiz nach einer Tibetischen Rezeptur hergestellte Präparat wirkt auf verschiedenen Ebenen der Atherogenese. So hat es neben der anti-entzündlichen und immunmodulierenden Wirkung [10,11] auch anti- und prooxidativen Charakter [8].

In diesem Zusammenhang stellte sich die Frage, ob das Vielstoffgemisch auch einen therapeutisch relevanten Einfluss auf die Oxidierbarkeit von Blutlipiden hat und insbesondere LDLCholesterin vor Oxidation schützen kann. BRUNNER et al. [1] untersuchten in einer placebo-kontrollierten Doppelblind-Studie den Einfluss von PADMA 28 auf die Blutlipid-Oxidierbarkeit in vivo und in vitro.

Die Blutlipid-Oxidierbarkeit von 60 Probanden zwischen 30 und 65 Jahren mit mässig erhöhtem GesamtCholesterinspiegel und ohne klinische Anzeichen einer Arteriosklerose wurde in einer placebo-kontrollierten Doppelblind-Studie untersucht. Die Medikation bzw. die Placebogabe erfolgte mit $3 \times 2$ Kapseln pro Tag. Neben der LipidOxidierbarkeit ex vivo wurden die Lipidwerte (Total-, HDL- und LDL-Cholesterin sowie Triglyceride, Apo-Lipoprotein A1 und Apo-Lipoprotein B) bestimmt. Alle Werte wurden vor und nach einer 4-wöchigen Einnahme von PADMA 28 bzw. Placebo und zusätzlich eine Woche nach der letzten Einnahme gemessen. In vitro wurden isoliertes LDL und Plasma, beide unbehandelt oder vorinkubiert mit PADMA-28-Extrakt, mit dem Radikalgenerator AAPH oxidiert. Diese in vitro-Experimente bestätigten die Lipid-Peroxidation in Gegenwart von PADMA 28-Extrakt.

Im klinischen Versuch zeigte sich, dass nach der 4-wöchigen Einnahme von PADMA 28 die Blutlipid-Oxidierbarkeit signifikant gesenkt wurde (Abb. 1). Dieser Effekt war auch bei einer Kontrolle in der 5. Woche nachweisbar. In der Placebo-Gruppe veränderte sich dagegen die Lipid-Oxidierbarkeit über den gesamten Messzeitraum kaum (Abb. 2).

Die Werte von Apo-Lipoprotein A1 und Apo-Lipoprotein B zeigten keinen Unterschied zwischen der Verum- und der Placebo-Gruppe und veränderten sich über die ganze Messdauer nicht, ebenso blieb die primär nicht pathologische Endothelfunktion unverändert.

\section{Résumé}

Für eine umfassend wirksame Langzeitprävention und Behandlung von arteriosklerotischen Veränderungen ist eine Therapie erforderlich, die den chronischen Entzündungsprozess unterbricht, die Blutlipide vor Oxidation schützt und immunmodulierend wirkt.

Wie in verschiedenen Studien nachgewiesen werden konnte, moduliert PADMA 28 die unterschiedlichen Entwicklungsstufen der Atherogenese positiv. So wird die Produktion von E-Selektin vermindert und dadurch die Anhaftung der Monozyten an das Endothel unterbunden [9]. Die Bildung von p ro-inflammatorischen Zytokinen durch T-Helferzellen Typ 1 (Th1) und die Aktivierung von Makrophagen wird gehemmt [10,11]. Durch die vermehrte Produktion von Hämoxigenase-1 wird die Proliferation glatter Muskelzellen unterdrückt, die zur Bildung der «Fibrous Cap» und Stenosierung der Gefässe beiträgt [9].

BRUNNER et al. [1] konnten die bisher in vitro festgestellte anti-oxidative Wirkung von PADMA 28 bestätigen [8] und sowohl in vitro als auch in vivo beweisen, dass PADMA 28 Blutlipide, insbesondere LDL-Cholesterin vor Oxidation schützen kann.

\section{Literatur}

1. Brunner-La Rocca, H.P. Schindler,R., Schlumpf. M., Saller, R., Suter, M.: Effects of Tibetan herbal preparation PADMA 28 on blood lipids and lipid oxidisability in sujects with mild hypercholesterolaemia, VASA 2005;34:11-17.

2. Binder, Chr. et al: Innate and aquired immunity in atherogenesis, Nature Medicine Vol. 8, Nr. 11, November 2002

3. Biedermann, B.C. Arterioskleros- Eine Autoimmunkrankheit? Praxis 2003;92:1781-1783.

4. Manuskripte des Wissenschaftlichen Symposium anlässlich des 10-jährigen Bestehens der Deutschen Gesellschaft zur Bekämpfung von Fettstoffwechselstönngen und ihren Folgeerkrankungen DGFF (Lipid-Liga) e.V. am 17.10.1998 in Wiesbaden: H.U. Klör, Medizinische Klinik und Poliklinik der Justus Liebig Universität Giessen.

5. Manuskripte des Wissenschaftlichen Symposium anlässlich des 10 jährigen Bestehens der Deutschen Gesellschaft zur Bekämpfung von Fettstoffwechselstörungen und ihren Folgeerkrankungen DGFF (Lipid-Liga) e.V. am 17.10.1998 in Wiesbaden: A. Weizel, Heinrich Lanz Krankenhaus Mannheim.

6. Landmesser, U. et al. Oxidant Stress as a Marker for Cardiovascular Events Ox Marks the Spot Circulation 2001;104:2638-2640.

7. Shoenfeld, Y. et al. Atherosclerosis as an infectious, inflammatory and autoimmune disease. Research Update TRENDS in Immunology Vol.22, No.6, June 2001.

8. Suter M, Richter Ch. Anti- and pro-oxidative properties of PADMA 28, a Tibetan herbal formula. Redox Rep 2000;5(1):17-22.

9. Exner, M. Antiinflammatorische Mechanismen des Tibetischen Arzneimittels Padma 28: Die Rolle von E-Selektin und Hämoxygenase-1. 3. Luzerner Phytotherapiegespräch 2003.

10. Barak, V. Padma 28, A Tibetan herbal preparation is an inhibitor of inflammatory cytokine p roduction. Eur. Cytokine Netw., Vol. 15, No 3, September 2004, 203-209.

11. Neurauter et al. Padma 28 modulates interferon- $\gamma$-induced tryptophan degradation and neopterin production in human PBMC in vitro. International Immunopharmacology 4 (2004) 833-839.

12. Boaz M, Smetana $S$, Weinstein $T$, Matas $Z$, Gafter U, laina A, Knecht A, Weissgarten $Y$, Brunner D, Fainaru M, Green MS. Secondary prevention with antioxidants of cardiovascular disease in endstage renal disease (SPACE): randomised placebo-controlled trial. Lancet. 2000 Oct 7;356(9237):1213-8.

13. Ryglewicz D, Rodo M, Roszczynko M, Baranska-Gieruszczak M, Szirkowiec W, Swiderska $\mathrm{M}$, Wehr H. Dynamics of LDL oxidation in ischemic stroke patients. Acta Neurol Scand. 2002 Mar;105(3):185-8.

14. Andrews B, Burnand $K$, Paganga G, Browse N, Rice-Evans C, Sommerville K, Leake D, Taub N. Oxidisability of low density lipoproteins in patients with carotid or femoral artery atherosclerosis. Atherosclerosis. 1995 Jan 6; 112(1):77-84.

15. Vassalle $C$, Petrozzi L, Botto N, Andreassi MG, Zucchelli GC. Oxidative stress and its association with coronary artery disease and different atherogenic risk factors. J Intern Med. 2004 Oct;256(4):308-15.

\section{Anschrift der Autorin:}

Dr. med. Margarethe Combé

Peter Merian-Strasse 58, CH-4002 Basel 